873

\section{PHARMACOLOGIC MACROPHAGE DEPLETION AFFECTS METASTASIS FORMATION BY MODULATING SYSTEMIC IMMUNE RESPONSES IN A GENETIC PANCREATIC CANCER MODEL}

${ }^{1}$ Heidi Griesmann*, ${ }^{1}$ Heidi Griesmann, ${ }^{1}$ Heidi Griesmann, ${ }^{2}$ Christof Drexel, ${ }^{2}$ Nada Milosevic, ${ }^{3}$ Bence Sipos, ${ }^{2}$ Thomas Gress, ${ }^{1}$ Patrick Michl. ${ }^{1}$ University Halle, Halle, Germany; ${ }^{2}$ University Marburg, Marburg, Germany; ${ }^{3}$ University Tuebingen, Tuebingen, Germany

Background Tumour-associated macrophages (TAM) play an important role in mediating tumour progression. In pancreatic cancer, infiltrating macrophages have been identified not only in invasive tumours, but also in early preinvasive pancreatic intraepithelial neoplasias and are known to mediate tumour progression.

Methods We aimed to study the impact of pharmacological macrophage depletion by liposomal clodronate in the genetic mouse model of pancreatic cancer (KPC mouse: LSLKrasG12D/+;LSL-Trp53R172H/+;Pdx-1-Cre). KPC mice were treated with liposomal clodronate or control liposomes from week 8 to week 20. Tumour and metastasis formation as well as alterations in local and circulating immune cells and cytokines were analysed.
Results Treatment with liposomal clodronate effectively reduced CD11b-positive macrophages both in the pancreas and other organs such as liver, lung and spleen. Tumour incidence and size was only slightly reduced. However, metastasis formation in the liver und lungs was markedly diminished after macrophage depletion. Reduced macrophage count was associated with significant alterations in circulating growth factors and mediators known to be secreted by macrophages and associated with angiogenesis, most prominently VEGF. Moreover, application of liposomal clodronate led to marked alterations in circulating immune cells, among them reduced regulatory $\mathrm{T}$ cells.

Conclusions Pharmacological depletion of macrophages in a genetic mouse model of pancreatic cancer markedly reduced metastasis formation and is associated with modulated profile of both secreted mediators and regulatory $\mathrm{T}$ cells. Pharmacological modulation of infiltrating macrophages represents a promising avenue for antimetastatic therapeutic approaches.

http://dx.doi.org/10.1136/jitc-2020-SITC2020.0873 